\title{
Public mental health: the local tangibles
}

\author{
Jonathan Campion ${ }^{1}$
}

The Psychiatrist (2013), 37, 238-243, doi: 10.1192/pb.bp.113.042788

${ }^{1}$ South London and Maudsley NHS Foundation Trust, London

Correspondence to Jonathan Campion (Jonathan.Campion@slam.nhs.uk) First received 16 Jan 2013, final revision 27 Mar 2013, accepted 3 Apr 2013
Summary Public mental health involves a number of important elements relevant to psychiatrists. This includes estimation of the level and impact of unmet need for treatment of mental disorder, prevention of mental disorder and promotion of mental well-being particularly in higher-risk groups. In the UK, the majority of people with mental disorder receive no intervention. This is despite the large impact and costs of mental disorder and existence of cost-effective interventions which result in economic savings even in the short term. Furthermore, spend is virtually absent for interventions to prevent mental disorder and promote mental well-being despite prominence within government policy and a good evidence base. At a time of budget cuts, local information about level, impact and costs of such unmet need as well as effective interventions are important to accurately inform the required investment for treatment of mental disorder, its prevention and promotion of mental well-being. Information about impact of interventions including economic savings across a broad range of sectors can also be estimated. This article summarises recent public mental health commissioning guidance which provides such information.

\section{What is public mental health?}

Public mental health involves: ${ }^{1}$

- assessment of levels of mental disorder and well-being, risk and protective factors in the local population including those from higher-risk groups

- enabling delivery of appropriate level of interventions to treat mental disorder early, prevent mental disorder and promote mental well-being

- ensuring that people at higher risk of mental disorder and poor mental well-being are proportionately prioritised in assessment and intervention delivery.

The cross-government public mental health strategy, Confident Communities, Brighter Futures, ${ }^{2}$ describes a range of effective public mental health interventions. Both the current and previous cross-government mental health strategies $^{3,4}$ outline a twin-track approach, which includes early intervention for mental disorder and promotion of population mental well-being. One of the reasons for such an approach is the recognition that even if all those with mental disorder received the best available treatment, this would only partially alleviate the burden of mental disorder.

The Royal College of Psychiatrists' position statement on public mental health ${ }^{5}$ highlights the important public mental health role of psychiatrists. Many psychiatrists already adopt a public mental health approach in their work, although the position statement states that they should be engaged in the commissioning process and informing commissioners of the expected prevalence of mental disorders to anticipate levels of required service provision and unmet need. ${ }^{5}$ The College's position statement also advises that psychiatrists need to be engaged in assessing the promotion needs of their local population.

The government's public mental health White Paper ${ }^{6}$ signalled a new approach to public health, which places mental health and well-being at the heart of the new system, as an integral and complementary part of public health in England. Clinical commissioning groups and local authorities now jointly lead the local health and care system through health and well-being boards in collaboration with their communities. The boards have a key role in transforming health and care and achieving better population health and well-being through:

- joint strategic needs assessments (JSNAs), which bring together into one report the current and future health and social care needs of the local population to inform health and social care planning and commissioning ${ }^{7}$

- joint health and well-being strategies, which set out the local strategy and the priorities identified by the health and well-being board to tackle the needs identified in their JSNAs, which therefore require accurate information about level of unmet mental health need.

\section{Public mental health intelligence relevant to psychiatrists}

The following four areas of public mental health intelligence are relevant to both psychiatrists and JSNAs:

1 local levels of mental disorder and well-being, including in higher-risk groups

2 local levels of risk and protective factors 
3 impact of mental disorder and poor well-being

4 and proportion of the population receiving public mental health interventions, including those with mental disorder.

\section{Local levels of mental disorder and well-being including in higher-risk groups}

One in two people experience a mental disorder during their lifetime. ${ }^{8}$ In England, national psychiatric morbidity surveys show that mental disorder is common:

- $10 \%$ of children and young people experience a mental disorder ${ }^{9}$

- $17.6 \%$ of adults experience at least one common mental disorder ${ }^{10}$

- $0.4 \%$ of adults have psychosis ${ }^{10}$

- $6 \%$ have alcohol dependence ${ }^{10}$

- $3 \%$ are dependent on illegal drugs ${ }^{10}$

- $21 \%$ have tobacco dependence ${ }^{11}$

- $5.4 \%$ of men and $3.4 \%$ of women have diagnosable personality disorder ${ }^{12}$

- $5 \%$ of people aged over 65 and $20 \%$ of those aged over 80 have dementia. ${ }^{13}$

Subthreshold mental disorders are also common, result in significant burden and increase the risk of transition to threshold disorder. Levels of subthreshold disorder include:

- $18 \%$ of 5 - to 16-year-olds experience subthreshold conduct disorder ${ }^{14}$

- $17 \%$ of adults experience subthreshold common mental disorder ${ }^{10}$

- $5 \%$ of adults experience subthreshold psychosis ${ }^{15}$

- $24 \%$ of adults drink more than the safe upper limit of alcohol. ${ }^{10}$

Well-being levels in the UK have been highlighted by an Office for National Statistics survey ${ }^{16}$ of 165000 adults, which found that for life satisfaction $76 \%$ scored 7 or more out of 10 and $7 \%$ scored less than 5, for life being worthwhile $80 \%$ scored 7 or more and $5 \%$ scored less than 5 , whereas for happiness yesterday $78 \%$ scored 7 or more and $11 \%$ scored less than 5 . An important risk factor for poor well-being affecting a large proportion of the population is mental disorder and subthreshold mental disorder.

\section{Local variation of mental disorder and well-being}

Although information about national levels of mental disorder and well-being is helpful, significant local variation occurs. ${ }^{10,17}$ Such variation is due to differences in local levels of risk and protective factors as well as variation in numbers from higher-risk groups. This highlights the importance of accurate assessment of local levels of mental disorder and well-being to inform about numbers requiring intervention and about the level of unmet need. ${ }^{1}$

Levels of mental disorder and well-being in higher-risk groups Certain groups at much higher risk of mental disorder are also likely to have lower levels of well-being and disproportionately benefit from public mental health interventions. Therefore, information is required about both the local numbers from such groups and their level of increased risk. ${ }^{6}$ Examples of child and adolescent groups with several-fold increased risk include children with intellectual disability, special education needs, physical illness, with a parent with mental disorder, looked-after children and young offenders. ${ }^{1}$ Examples of adult higherrisk groups include certain Black and minority ethnic groups, homeless people, prisoners, and lesbian, gay, bisexual and transsexual people.

\section{Local levels of risk and protective factors}

Mental disorder and well-being are associated with a range of risk and protective factors. ${ }^{1,18}$ A public health approach emphasises the importance of addressing such factors to prevent mental disorder and promote well-being. Commissioning decisions can be informed by local level of risk and protective factors including in higher-risk groups as well as impact of such factors. ${ }^{1}$

Factors associated with increased risk of mental disorder during childhood and adolescence include: ${ }^{1}$

- during pregnancy and at birth:

- smoking during pregnancy is associated with increased risk of conduct disorder ${ }^{19}$

- alcohol consumption during pregnancy: fetal alcohol spectrum disorder is the most common non-genetic cause of intellectual disability ${ }^{20}$

- stress: maternal stress during pregnancy is associated with child behavioural problems ${ }^{21}$ and impaired cognitive and language development ${ }^{22}$

- premature birth is associated with increased risk of mental illness in offspring ${ }^{23}$

- women with mental disorder have an increased risk of low birth weight and prematurity in their offspring ${ }^{24,25}$

- socioeconomic factors including household income, state benefits, tenure and type of area: ${ }^{9}$ children from lowest household income have threefold increased risk of mental disorder ${ }^{9}$

- family factors including family structure ${ }^{9}$

- parental factors: education qualifications, employment and presence of mental disorder ${ }^{9}$

- child factors: age, gender, ethnicity, special education needs and physical illness.

Some of the strongest predictors of mental disorder are childhood adversity, particularly when associated with maladaptive family functioning (such as parental mental disorder, child abuse, neglect) ${ }^{26}$ For instance, child abuse is associated with increased risk of depression (odds ratio $(\mathrm{OR})=2.9)$, post-traumatic stress disorder (PTSD) $(\mathrm{OR}=4.0)$, psychosis $(\mathrm{OR}=2.7)$, alcohol dependence $(\mathrm{OR}=1.8)$ and drug problems $(\mathrm{OR}=2.1){ }^{27}$ Repeated sexual abuse during childhood and adolescence is associated with even higher rates of adult mental disorder including depressive disorder $(\mathrm{OR}=6.2)$, PTSD $(\mathrm{OR}=6.8)$, probable psychosis $(\mathrm{OR}=15.3)$, alcohol dependence $(\mathrm{OR}=5.2)$, eating disorder $(\mathrm{OR}=11.7)^{27}$ and attempted suicide $(\mathrm{OR}=9.4) .^{28}$

Factors associated with mental disorder in adulthood include deprivation, unemployment, debt, violence, stressful life events, inadequate housing and fuel poverty. ${ }^{1}$ Factors associated with dementia include smoking, hypertension and high body mass index. 
Inequality and deprivation are key underlying elements for a range of risk factors for mental disorder and poor mental well-being, which then further increase inequality. Whereas higher-risk groups benefit more from public mental health interventions, larger groups at less elevated risk also benefit. Therefore, a proportionate universalistic approach can both target higher-risk groups and accommodate larger population groups.

\section{Population level of risk factors}

As well as level of increased risk associated with particular factors, it is also important to assess the local level of such risk factors. ${ }^{1,18}$ For instance, $25.3 \%$ of 18 - to 24 -year-olds and $18.6 \%$ of 11 - to 17-year-olds experienced severe maltreatment during childhood. Similarly, $2.9 \%$ of women and $0.8 \%$ of men experienced sexual abuse in childhood (sexual intercourse), whereas $11.1 \%$ of women and $5.3 \%$ of men experienced sexual touching in childhood. ${ }^{1}$ The Guidance for Commissioning Public Mental Health Services ${ }^{1}$ also provides information on how to accurately estimate overall numbers of people in different higher-risk groups who have a mental disorder.

\section{Impact of mental disorder and poor well-being}

In the UK, mental disorder is responsible for $22.8 \%$ of burden of disease; for comparison, the figure for cardiovascular disease is $16.2 \%$ and for cancer $15.9 \% .^{29}$ No other health condition matches mental ill health in the combined extent of prevalence, persistence and breadth of impact. It is therefore important that local strategy development, commissioning and public health take account of the local impact of mental disorder.

A key reason for the size of impact is that mental disorder starts early, with $50 \%$ of lifetime mental illness (excluding dementia) starting by age $14,{ }^{8,30}$ and $75 \%$ starting by the mid-20s. ${ }^{31}$ Mental disorder during childhood and adolescence results in a broad range of disadvantages including worse health, social skills and school outcomes, higher levels of health-risk behaviours such as smoking, alcohol and drug misuse, higher levels of self-harm and suicide, as well as antisocial behaviour and offending (Table 1). ${ }^{1,18}$

Mental disorder during childhood and adolescence also results in worse subsequent adult health outcomes including higher rates of adult mental disorder and suicide, unemployment and lower earnings, marital problems, crime and violence. ${ }^{1}$ In adults, mental disorder increases risk of:

- physical illness

- reduced life expectancy - for instance, schizophrenia is associated with 20.5 years' reduced life expectancy for men and 16.4 years' reduced life expectancy for women $^{32}$

- suicide and self-harm

- health-risk behaviour including poor diet, less exercise, more smoking, drug and alcohol misuse; smoking is the largest single preventable cause of death, yet $42 \%$ of adult tobacco consumption in England is by those with mental disorder ${ }^{11}$ and $43 \%$ of under-17-year-old smokers have either emotional or conduct disorder ${ }^{9}$

- unemployment
- poor housing

- stigma and discrimination.

\section{Economic impact of mental disorder}

National costs of mental disorder which arise from this broad range of factors are as follows (such costs can also be calculated locally):

- to economy: $£ 105.2$ billion annual cost of mental health problems in England ${ }^{33}$

- to the National Health Service (NHS): $£ 11.9$ billion or $11.1 \%$ of annual budget spent on mental health services in $2009 / 2010^{34}$

- to UK employers: $£ 28.3$ billion annually ${ }^{35}$

- crime: $£ 60$ billion annual cost of crime in England and Wales by adults who had conduct problems during childhood and adolescence. ${ }^{36}$

\section{Benefits of mental well-being}

The benefits of well-being are due to more than just absence of mental disorder. ${ }^{5}$ Health benefits of mental well-being include reductions in: mental disorder in children and adolescents, including persistence; mental disorder and suicide in adults; physical illness; associated healthcare utilisation; and mortality. ${ }^{1,18}$ Benefits of mental well-being outside health include improved educational outcomes, healthier lifestyle, reduced risk-taking, increased productivity at work, fewer missed days off work, higher income, better social relationships, reduced antisocial behaviour, crime and violence, and reduced substance misuse.

\section{Numbers receiving intervention to treat mental disorder, prevent mental disorder and promote mental health: estimating level of unmet need}

Regarding treatment, the most recent national psychiatric morbidity surveys show that only a minority of those in the UK with mental disorder except psychosis receive any intervention; ${ }^{9,10}$ the following proportion receiving any intervention for different mental disorder:

- $23-28 \%$ of children with emotional and conduct disorders are seen by child and adolescent mental health services and receive less support from other services

- $24 \%$ of adults with common mental disorder; however, less than $10 \%$ of older people with depression receive adequate treatment

- $28 \%$ of adults screening positive for PTSD

- $81 \%$ of adults with probable psychosis received some form of treatment compared with $85 \%$ in 2000

- $65 \%$ of adults with 'psychotic disorder' in past year

- $14 \%$ of adults with alcohol dependence

- $14 \%$ of adults with cannabis dependence

- $36 \%$ of adults with dependence on other drugs.

Public mental health commissioning guidance ${ }^{1}$ enables estimation of the local proportion of those with different mental disorder who receive intervention, thereby highlighting the size of unmet need. The guidance also draws attention to the fact that an even smaller proportion receive prevention or promotion interventions and highlights ways to estimate local promotion and prevention of unmet need. Establishing the size of unmet need informs the JSNA, 


\begin{tabular}{|c|c|c|c|c|}
\hline & $\begin{array}{l}\text { Emotional } \\
\text { disorder, \% }\end{array}$ & $\begin{array}{l}\text { Conduct } \\
\text { disorder, \% }\end{array}$ & $\begin{array}{l}\text { Hyperkinetic } \\
\text { disorder, \% }\end{array}$ & $\begin{array}{l}\text { Whole survey } \\
\text { prevalence, \% }\end{array}$ \\
\hline \multicolumn{5}{|l|}{ Age $5-16$ years } \\
\hline \multicolumn{5}{|l|}{ Health and social skill outcomes } \\
\hline Health is fair or bad (parent report) & 23 & 17 & 18 & 7 \\
\hline Found it harder than average to make friends & 35 & 24 & 32 & 10 \\
\hline No friends & 6 & 8 & 10 & 2 \\
\hline \multicolumn{5}{|l|}{ School outcomes } \\
\hline 2 or more years behind in intellectual development & 23 & 36 & 40 & 9 \\
\hline More than 15 days' absence in the previous term & 17 & 14 & 11 & 4 \\
\hline Ever been excluded from school & 12 & 34 & 29 & 2 \\
\hline \multicolumn{5}{|l|}{ Age $11-16$ years } \\
\hline $\begin{array}{l}\text { Self-reported risk-taking behaviours } \\
\text { Regularly smokes }\end{array}$ & 19 & 30 & 15 & 6 \\
\hline Regularly drinks & 13 & 19 & 13 & 9 \\
\hline Drinks twice a week or more & 5 & 12 & 7 & 3 \\
\hline Taken drugs at some time & 20 & 28 & 23 & 8 \\
\hline Taken drugs, mainly cannabis & 16 & 23 & 18 & 7 \\
\hline Taken drugs other than cannabis & 7 & 16 & 7 & 2 \\
\hline Self-reported self-harm & 28 & 21 & 18 & 7 \\
\hline
\end{tabular}

which in turn informs health and well-being board decisions regarding priorities and commissioning.

The proportion of individuals receiving public mental health interventions is related to available local resources. The commissioning guidance ${ }^{1}$ includes spend per head of population since level of local resource often does not reflect the level of mental disorder and poor well-being.

The previous section on public mental health intelligence can support coordinated delivery of effective public mental health intervention.

Delivery of effective public mental health interventions A range of effective public mental health interventions to treat mental disorder early, prevent mental disorder and promote well-being are outlined in several policy and other publications. ${ }^{2-6,18}$

Early intervention for mental disorder is associated with improved outcomes, but it can also prevent a significant proportion of adult mental disorder ${ }^{30}$ and associated wide-ranging consequences. Early promotion of recovery through activities such as supported employment, housing assistance and debt advice is also important. Early promotion of physical health, prevention of health-risk behaviour and detection of physical illness can defend against the significantly reduced life expectancy in those who develop a mental disorder. ${ }^{1}$

One of the reasons why only a minority of people with a mental disorder receive any intervention is poor recognition by both the general population and health staff. Early recognition and intervention for mental disorder can be facilitated by both health staff training and wider population campaigns to highlight symptoms, similar to campaigns for other health issues, such as cancer. Regarding prevention, although early intervention for mental disorder, associated health risk behaviour and physical health can prevent a range of outcomes, a number of interventions can prevent mental disorder and suicide. ${ }^{1}$ These include interventions to address inequalities, stigma, discrimination, violence and abuse, whereas others can prevent a range of health risk behaviours such as smoking. In addition, a range of effective interventions can promote well-being across the life course. ${ }^{1}$

Public mental health interventions are delivered by a range of service providers including primary and secondary care, social care, public health, local authorities, third sector, education, employers and criminal justice. Therefore, public mental health also has an important role in facilitating greater collaboration and coordination of activities of such a group of organisations. A coordinated delivery of public mental health interventions results not only in improvement in a range of outcomes, but also in economic savings, reduced inequalities and parity of esteem.

Due to the wide impact of mental disorder and wellbeing, public mental health interventions improve a range of NHS, public health and social care outcomes, ${ }^{1}$ resulting in improved mental health, physical health, life expectancy, healthy lifestyles, resilience, economic productivity, social functioning and quality of life. They also reduce the burden and economic costs of mental ill health, health-risk behaviours, crime and violence.

\section{Economic savings}

The London School of Economics and Political Science estimated the significant economic savings which arise from public mental health interventions even in the short term as part of the mental health strategy. ${ }^{37}$ This work highlighted where savings occur and stressed that lack of intervention results in higher economic cost. For early intervention of mental disorder, each pound spent results in the following net savings sometimes within the first year: ${ }^{37}$

- parenting interventions for families with conduct disorder (£8)

- early diagnosis and treatment of depression at work (£5)

- early intervention of psychosis (£18)

- early intervention for psychosis at-risk mental state (£10)

- screening and brief interventions in primary care for alcohol misuse (£12). 
For mental health promotion, each pound results in the following net savings: ${ }^{37}$

- school-based social and emotional learning programmes (£84)

- school-based interventions to reduce bullying (£14)

- work-based mental health promotion (£10 with savings in year 1)

- debt advice services (£4).

Targeted promotion interventions for those recovering from mental illness result in the following savings: ${ }^{1}$

- employment support (individual placement support) for people with severe mental illness: annual savings of $£ 6000$ per client

- housing support services for men with enduring mental illness: annual savings £11 000-£20000 per client.

The commissioning guidance ${ }^{1}$ outlines how local economic savings of such interventions can be calculated depending on the proportion receiving intervention. This can enhance investment.

\section{Reduced inequalities and parity of esteem}

As highlighted in the section on prevention, interventions to address inequality can also prevent mental disorder. ${ }^{1}$ Mental disorder itself results in a further range of inequalities which can also be prevented by early treatment of mental disorder, early intervention for health-risk behaviours, early treatment of physical illness and early targeted promotion of well-being to facilitate recovery.

Only a minority of people with mental disorder in the UK receive any intervention, ${ }^{9,10}$ in contrast to conditions such as cancer where almost all affected receive intervention. Those with mental disorder also have reduced access to interventions for health-risk behaviour and treatment of physical illness compared with the general population. Almost no spend occurs on cost-effective interventions to prevent mental disorder and promote mental well-being despite its prominence in mental health and public health policy. ${ }^{34}$ Such a level of inaction has a broad range of impacts and costs affecting a large proportion of the population and may be due to a combination of lack of public mental health knowledge and discriminating attitudes towards mental health. Transparency about the local impact of mental disorder, proportion receiving intervention and the effects of inaction can facilitate parity and investment.

\section{Conclusions}

Clinical commissioning groups and local authorities now jointly lead local healthcare systems through health and well-being boards in collaboration with their communities, ${ }^{7}$ whereas local authorities have also taken on responsibility for public health. Significant local variation exists in levels of mental disorder, well-being and public mental health interventions. The JSNA is a key vehicle for highlighting local relevant public mental health information, although this is often missing. The commissioning guidance ${ }^{1}$ enables inclusion of local public mental health information such as level of risk and protective factors, numbers from groups at higher risk of mental disorder and poor well-being, local levels of mental disorder and well-being, and proportion receiving public mental health interventions, including those from higher-risk groups, which enables estimation of level of unmet need. Such information enables transparency about the proportion of the local population which commissioners consider acceptable to provide cost-effective public mental health interventions for. The local impact of such decisions including economic costs or savings can also be estimated.

\section{About the author}

Jonathan Campion is Director for Public Mental Health and Consultant Psychiatrist, South London and Maudsley NHS Foundation Trust, London, UK. He is Visiting Professor of Population Mental Health at University College London where he is Director of Population Mental Health for the UCLP Integrated Mental Health Programme.

\section{References}

1 Campion J, Fitch C. Guidance for Commissioning Public Mental Health Services. Joint Commissioning Panel for Mental Health, 2012.

2 Department of Health. Confident Communities, Brighter Futures: A Framework for Developing Well-Being. Department of Health, 2010

3 HM Government, Department of Health. No Health without Mental Health: A Cross-Government Mental Health Outcomes Strategy for People of All Ages. HM Government, 2011.

4 HM Government. New Horizons: Towards A Shared Vision for Mental Health. Department of Health, 2009.

5 Royal College of Psychiatrists. No Health without Public Mental Health: The Case for Action (Position Statement PS4/2010). Royal College of Psychiatrists, 2010.

6 HM Government. Healthy Lives, Healthy People: Our Strategy for Public Health in England. TSO (The Stationery Office), 2010.

7 Department of Health. Joint Strategic Needs Assessment and Joint Health and Wellbeing Strategies Explained. Department of Health, 2011.

8 Kessler RC, Berglund P, Demler O, Jin R, Merikangas KR, Walters EE. Lifetime prevalence and age-of-onset distributions of DSM-IV disorders in the National Comorbidity Survey Replication. Arch Gen Psychiatry 2005; 62: 593-602

9 Green H, McGinnity A, Meltzer H, Ford T, Goodman R. Mental Health of Children and Young People in Great Britain, 2004. Office for National Statistics, 2005

10 McManus S, Meltzer H, Brugha T, Bebbington P, Jenkins R (eds) Adult Psychiatric Morbidity in England, 2007: Results of a Household Survey. The NHS Information Centre, 2009.

11 McManus S, Meltzer H, Campion J. Cigarette Smoking and Mental Health in England: Data from the Adult Psychiatric Morbidity Survey 2007. National Centre for Social Research, 2010.

12 Singleton N, Bumpstead R, O'Brien M, Lee A, Meltzer H. Psychiatric Morbidity among Adults Living in Private Households, 2000. TSO (The Stationery Office), 2001.

13 Knapp M, Prince M. Dementia UK: A Report into the Prevalence and Cost of Dementia. Alzheimer's Society, 2007.

14 Colman I, Murray J, Abbott RA, Maughan B, Kuh D, Croudace TJ, et al. Outcomes of conduct problems in adolescence: 40 year follow-up of national cohort. BMJ 2009; 338: a2981.

15 van Os J, Linscott RJ, Myin-Germeys P, Delespaul P, Krabbendam L. A systematic review and meta-analysis of the psychosis continuum: evidence for a psychosis proneness-persistence-impairment model of psychotic disorder. Psychol Med 2009; 39: 179-95.

16 Office for National Statistics. First ONS Annual Experimental Subjective Well-being Results. ONS, 2012. 
17 Deacon L, Carlin H, Spalding J, Giles S, Stansfield J, Hughes S, et al. North West Mental Wellbeing Survey 2009. North West Public Health Observatory, 2009

18 Campion J, Bhui K, Bhugra D. European Psychiatric Association (EPA) guidance on prevention of mental disorders. Eur Psychiatry 2012; 27 68-80.

19 Murray J, Irving B, Farrington DP, Colman I, Bloxsom CA. Very early predictors of conduct problems and crime: results from a national cohort study. J Child Psychol Psychiatry 2010; 51: 1198-207.

20 Carpenter B. Pedagogically bereft! Improving learning outcomes for children with foetal alcohol spectrum disorders. Br J Spec Educ 2011; 38 37-43.

21 O'Connor TG, Heron J, Golding J, Glover V. Maternal antenatal anxiety and behavioural/emotional problems in children: a test of a programming hypothesis. J Child Psychol Psychiatry 2003; 44: 1025-36.

22 Bergman K, Sarkar P, O'Connor TG, Modi N, Glover V. Maternal stress during pregnancy predicts cognitive ability and fearfulness in infancy. J Am Acad Child Adolesc Psychiatry 2007; 46: 1454-63.

23 Nosarti C, Reichenberg A, Murray RM, Cnattingius S, Lambe MP, Yin L, et al. Preterm birth and psychiatric disorders in young adult life. Arch Gen Psychiatry 2012; 69: 610-7.

24 National Institute for Health and Clinical Excellence. Antenatal and Postnatal Mental Health: Clinical Management and Service Guidance (Clinical Guideline CG45). NICE, 2007.

25 Grote NK, Bridge JA, Gavin AR, Melville JL, Ivengar S, Katon WJ. A meta-analysis of depression during pregnancy and the risk of preterm birth, low birth weight, and intrauterine growth restriction. Arch Gen Psychiatry 2010; 67: 1012-24.

26 Kessler RC, McLaughlin KA, Green JG, Gruber MJ, Sampson NA Zaslavsky AM, et al. Childhood adversities and adult psychopathology in the WHO World Mental Health Surveys. Br J Psychiatry 2010; 197: $378-85$.
27 Jonas S, Bebbington P, McManus S, Meltzer H, Jenkins R, Kuipers E, et al. Sexual abuse and psychiatric disorder in England: results from the 2007 Adult Psychiatric Morbidity Survey. Psychol Med 2011; 41 709-19.

28 Bebbington PE, Cooper C, Minot S, Brugha TS, Jenkins R, Meltzer H, et al. Suicide attempts, gender and sexual abuse: data from the 2000 British Psychiatric Morbidity Survey. Am J Psychiatry 2009; 166: 113540

29 World Health Organization. The Global Burden of Disease: 2004 Update. WHO, 2008.

30 Kim-Cohen J, Caspi A, Moffitt TE, Harrington H, Milne BJ, Poulton R. Prior juvenile diagnoses in adults with mental disorder: developmental follow-back of a prospective longitudinal cohort. Arch Gen Psychiatry 2003; 60: 709-17.

31 Kessler RC, Amminger GP, Aguilar-Gaxiola S, Alonso J, Lee S, Ustün TB Age of onset of mental disorders: a review of recent literature. Curr Opin Psychiatry 2007; 20: 359-64.

32 Brown S, Kim M, Mitchell C, Inskip H. Twenty-five year mortality of a community cohort with schizophrenia. Br J Psychiatry 2010; 196: 116-21.

33 Centre for Mental Health. The Economic and Social Costs of Mental Health Problems in 2009/10. Centre for Mental Health, 2010.

34 Department of Health. Programme Budgeting Tools and Data: National Expenditure Data. Department of Health, 2012.

35 National Institute for Health and Clinical Excellence. Promoting Mental Wellbeing at Work: Full Guidance (Public Health Guidance PH22). NICE, 2009.

36 Sainsbury Centre for Mental Health. The Chance of a Lifetime: Preventing Early Conduct Problems and Reducing Crime. Sainsbury Centre for Mental Health, 2009.

37 Knapp M, McDaid D, Parsonage M (eds) Mental Health Promotion and Mental Illness Prevention: The Economic Case. Department of Health, 2011. 